\title{
Estimating the single-element concentration of intercalated insulators for the emergence of superconductivity
}

\author{
Shermane M. Benjamin* \\ Physics Department, Montana State University, Bozeman, MT 59717-3840, USA \\ E-mail: sbenjamin21@gmail.com
}

**Keep in mind Equations (1) and (2) were defined at the onset, not away from it. The analysis below supposes, at a minimum, Equation (2) describes transition temperatures at concentrations above onset plus an unknown perturbation. This approach approximately fits data for verified phase diagrams with oscillatory behavior in terms of intercalate valence electrons per unit cell volume.**

$$
\begin{gathered}
P V_{u n i t}=\left(x_{\text {onset }} N_{\text {val }}\right) * K_{B} T_{c-\text { exp.onset }} \\
T_{c-\text { calc }}=A_{s} *\left|\left(\frac{N_{e p} E_{e p}+N_{e e}\left(E_{e e}-E_{f e r m i}\right)}{N_{v a l} K_{B}}\right)\right|, \text { where } A_{s}=4.01 * 10^{-5}
\end{gathered}
$$




\section{$\mathrm{Cu}_{x} \mathrm{TiSe}_{2}$}

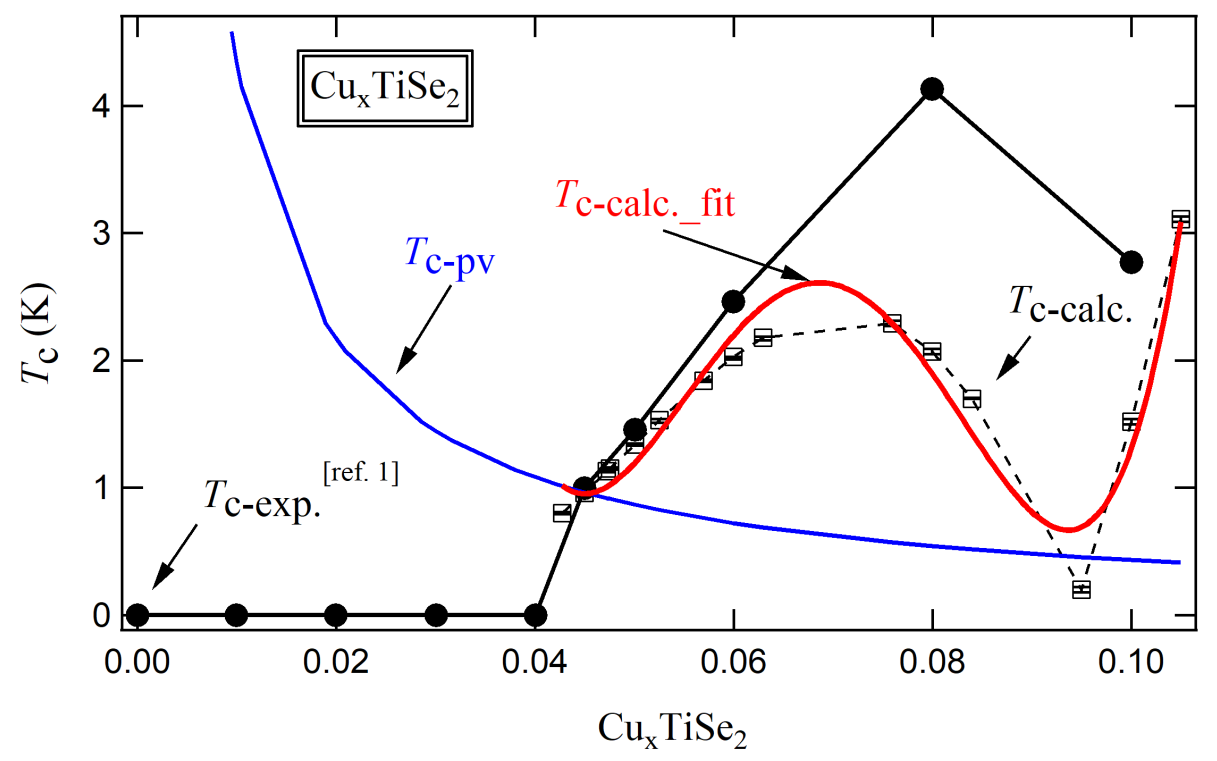

Figure S1: Transition temperature $\left(T_{c}\right)$ phase diagrams of superconducting and non-superconducting $\mathbf{C u}_{x} \mathbf{T i S e}_{2}$. Plots $T_{c-e x p}, T_{c-P V}, T_{c-c a l c}$, and $T_{c-c a l c-f i t}$ represent transition temperatures measured experimentally, ${ }^{1}$ estimated from equation (1), calculated from equation (2), and the least-squares fit of $T_{c-c a l c}$ with a 5 th order polynomial, respectively.

The experimental and calculated phase diagrams for $\mathrm{Cu}_{x} \mathrm{TiSe}_{2}$ are shown in Figure $\mathrm{S} 1$ as solid dots and open boxes, respectively. Constants found in the main report for $\mathrm{Cu}_{x} \mathrm{TiSe}_{2}$, including $N_{v a l}=11$ and lattice constants ${ }^{1} a=b=3.54 \AA$ and $c=6.01 \AA$, were used to calculate $T_{c-c a l c}$ from Equation (2). Experimental and calculated phase diagrams are comparable except near $x=0.8$.

Copper intercalated titanium diselenide, $\mathrm{Cu}_{x} \mathrm{TiSe}_{2}$, was first reported by Morosan et. al. ${ }^{1}$ as a series which contains superconducting phases at $0.045<x<0.1$. Thermal conductivity studies for $x=0.6$ show that it is a single-gap $s$-wave superconductor. ${ }^{2}$ The results from temperature dependent anisotropic magnetization and resistivity measurements for $x=0.7$ indicate that it is a BCS type- $I I$ superconductor. ${ }^{3}$ In general, angle-resolved photoemission spectroscopy show that the density of states is increased, which leads to superconductivity, 
when copper is intercalated. However, superconductivity vanishes with excessive copper intercalation because of strong inelastic scattering. ${ }^{4}$

\section{$\operatorname{Alkali}_{3} \mathbf{C}_{60}$}

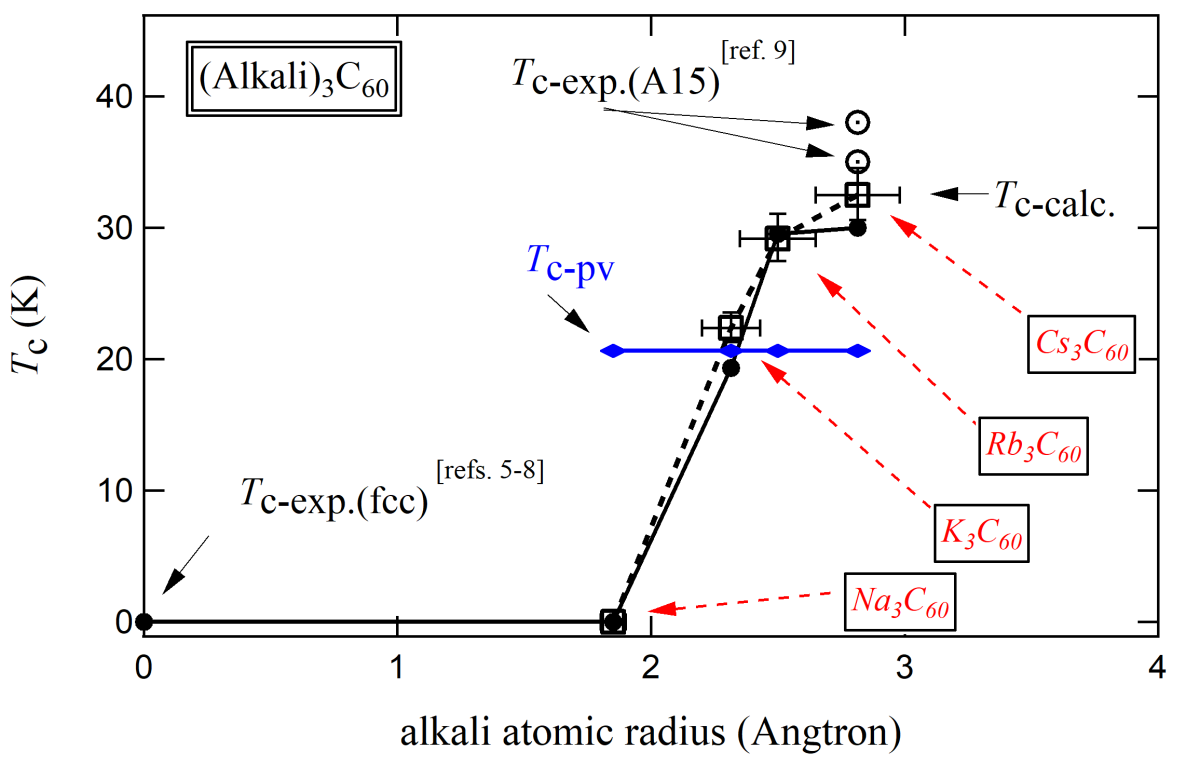

Figure S2: Transition temperature $\left(T_{c}\right)$ phase diagrams of superconducting and non-superconducting $\mathbf{A l k a l i}_{3} \mathbf{C}_{60}$. Plots $T_{c-\exp (f c c)}$ (black dots), $T_{c-\exp (A 15)}$ (open circles), $T_{c-P V}$ (blue line), and $T_{c-\text { calc }}$ (open boxes) represent transition temperatures measured experimentally for the f.c.c structure, ${ }^{5-8}$ measured experimentally for the A15 structure, ${ }^{9}$ estimated from equation (1) and calculated from equation (2), respectively.

The calculated transition temperature, $T_{c-c a l c}$, is in well agreement with data from experiment, $T_{c-\exp .(f c c)}$ and $T_{c-\exp .(A 15)}$. This may not be surprising as $x=1$ is very close to the onset for the compounds which exhibit superconductivity in this series. Open boxes represent calculated transition temperature $T_{\text {calc. }}$; error bars account for the difference between empirical ${ }^{10}$ and calculated ${ }^{11}$ atomic radii. There are at least 3 polymorphs of $\mathrm{Cs}_{3} \mathrm{C}_{60} \cdot{ }^{8}$ Black dots in Figure S2 represent data for the face centered cubic (f.c.c) structure, open circles represent the A15 structure. All f.c.c exhibit superconductivity at standard atmospheric pressure. The $\mathrm{A} 15$ of $\mathrm{Cs}_{3} \mathrm{C}_{60}$ requires a pressure of $P \approx 4.5$ kbar to generate the supercon- 
ducting state between $35 \mathrm{~K}$ and $38 \mathrm{~K} .{ }^{9}$ The calculated $T_{c}$ lies between the experimentally measured $T_{c}$ 's for both polymorphs of $\mathrm{Cs}_{3} \mathrm{C}_{60}$.

The Alkali $_{2+1} \mathrm{C}_{60}$ series, where Alkali $=\mathrm{Na}, \mathrm{K}, \mathrm{Rb}$, and $\mathrm{Cs}$, offer a unique opportunity to vary $R$ and $Z_{\text {eff }}$ simultaneously in Equation (2) as the concentration remains constant at $x=1$. Similar to other alkali intercalated insulators like $\mathrm{K}_{2+x} \mathrm{C}_{22} \mathrm{H}_{14}$ and $\mathrm{K}_{2+x} \mathrm{C}_{14} \mathrm{H}_{10}$, superconductivity first occurs at $x_{\text {exp.onset }}>0 .{ }^{12,13}$ More than likely it is because alkali metals tend to form dimers when introduced within a matrix, similar to $\mathrm{Rb}_{2}$ and $\mathrm{Cs}_{2}$ in solid helium for example. ${ }^{14,15}$ It has also been shown that $\mathrm{K}_{2} \mathrm{C}_{60}$ is a Mott-Hubbard insular that becomes metallic upon doping. ${ }^{16}$ For these reasons $\mathrm{Alkali}_{3} \mathrm{C}_{60}$ is rewritten as $\mathrm{Alkali}_{2+1} \mathrm{C}_{60}$ to emphasize $\mathrm{K}_{2} \mathrm{C}_{60}$ as the insulator that is being intercalated at $x=1$.

$$
P V_{u n i t}=\left.4.01 * 10^{-5} *\left(x N_{e p} E_{e p}+x N_{e e}\left[E_{e e}-E_{f e r m i}\right]\right)\right|_{x=1 \text { at } R_{m i n} \text { and } Z_{e f f-m i n}}
$$

To date, there exists 4 alkali intercalated buckminsterfullerenes $\left(\mathrm{C}_{60}\right)$. They are $\mathrm{Na}_{3} \mathrm{C}_{60},{ }^{5,17}$ $\mathrm{K}_{3} \mathrm{C}_{60},{ }^{6,18} \mathrm{Rb}_{3} \mathrm{C}_{60}{ }^{7}$ and $\mathrm{Cs}_{3} \mathrm{C}_{60} \cdot{ }^{8,9}$ The latter three superconduct at approximately $20 \mathrm{~K}, 30$ $\mathrm{K}$ and $38 \mathrm{~K}$, respectively. Sodium intercalated $\mathrm{C}_{60}$ does not superconduct. ${ }^{5}$ Equation (3) can be used to see that the onset of superconductivity occurs at $\mathrm{K}_{3} \mathrm{C}_{60}$. Using $\boldsymbol{P}=\mathbf{1 0 1}, \mathbf{3 2 5} \mathrm{Pa}$ and $\boldsymbol{V}_{\text {unit }}=\mathbf{2 8 1 0} \AA^{3}$, the left side of Equation $(3)$ is equal to $\mathbf{2 . 8 5} * \mathbf{1 0}^{\mathbf{- 2 2}} \mathrm{J}$. For the right side, with $\boldsymbol{N}_{e p}=\boldsymbol{N}_{e e}=\boldsymbol{N}_{\boldsymbol{v a l}}=\mathbf{1}, \boldsymbol{x}=\mathbf{1}$, and lattice parameters $\boldsymbol{a}=\boldsymbol{b}=\boldsymbol{c}=\mathbf{1 4 . 1 5}$ $\AA$, the compounds $\mathrm{Na}_{3} \mathrm{C}_{60}, \mathrm{~K}_{3} \mathrm{C}_{60}, \mathrm{Rb}_{3} \mathrm{C}_{60}$ and $\mathrm{Cs}_{3} \mathrm{C}_{60}$ yield $\mathbf{3 . 4} * \mathbf{1 0}^{-\mathbf{2 2}} \mathrm{J}$ (at $R=1.85 \AA$, $Z_{\text {eff }}=6.8018$ [for a $2 \mathrm{p}$ electron] $), \mathbf{3 . 0 9} * \mathbf{1 0}^{\mathbf{- 2 2}} \mathrm{J}$ (at $R=2.32 \AA, Z_{\text {eff }}=7.7256$ [for a $3 \mathrm{p}$ electron]), $\mathbf{4 . 0 3} * \mathbf{1 0}^{\mathbf{- 2 2}} \mathrm{J}$ (at $R=2.5 \AA, Z_{\text {eff }}=10.881$ [for a $4 \mathrm{p}$ electron]), and $\mathbf{4 . 4 8} * \mathbf{1 0}^{\mathbf{2 2}}$ $\mathrm{J}$ (at $R=2.82 \AA, Z_{\text {eff }}=13.651$ [for a $5 \mathrm{p}$ electron]), respectively. Notice outer-most $p$ electrons are used for $N_{e p}=1$ and calculating $E_{e p}$; this is because $N_{v a l}=N_{e e}=1$ for the outer-most $s$ electron for alkali elements. Clearly, $\mathrm{K}_{3} \mathrm{C}_{60}$ is the only compound that satisfies the minimum radius $R_{m i n}$ and minimum effective charge $Z_{\text {eff-min }}$ for Equation (3), within 
$10 \%$ accuracy. This means $\mathrm{K}_{3} \mathrm{C}_{60}$ represents the onset of superconductivity for alkali intercalated buckminsterfullerene within this framework when $x=1$; thus, the expected $T_{c}$ for $\mathrm{Na}_{3} \mathrm{C}_{60}$ must be zero since its $R$ and $Z_{\text {eff }}$ are smaller than that of $\mathrm{K}_{3} \mathrm{C}_{60}$. The blue curve of the "ideal" onset equation $\left(P V=x N K_{B} T_{c-P V}\right)$ shown in Figure S2 is a flat line that passes near $\mathrm{K}_{3} \mathrm{C}_{60}$ 's transition temperature as expected. The slope of $T_{c-P V}$ is zero because the "ideal" onset equation is independent of intercalate atomic radii. 


\section{$\mathrm{YBa}_{2} \mathrm{Cu}_{3} \mathrm{O}_{6+x}$}
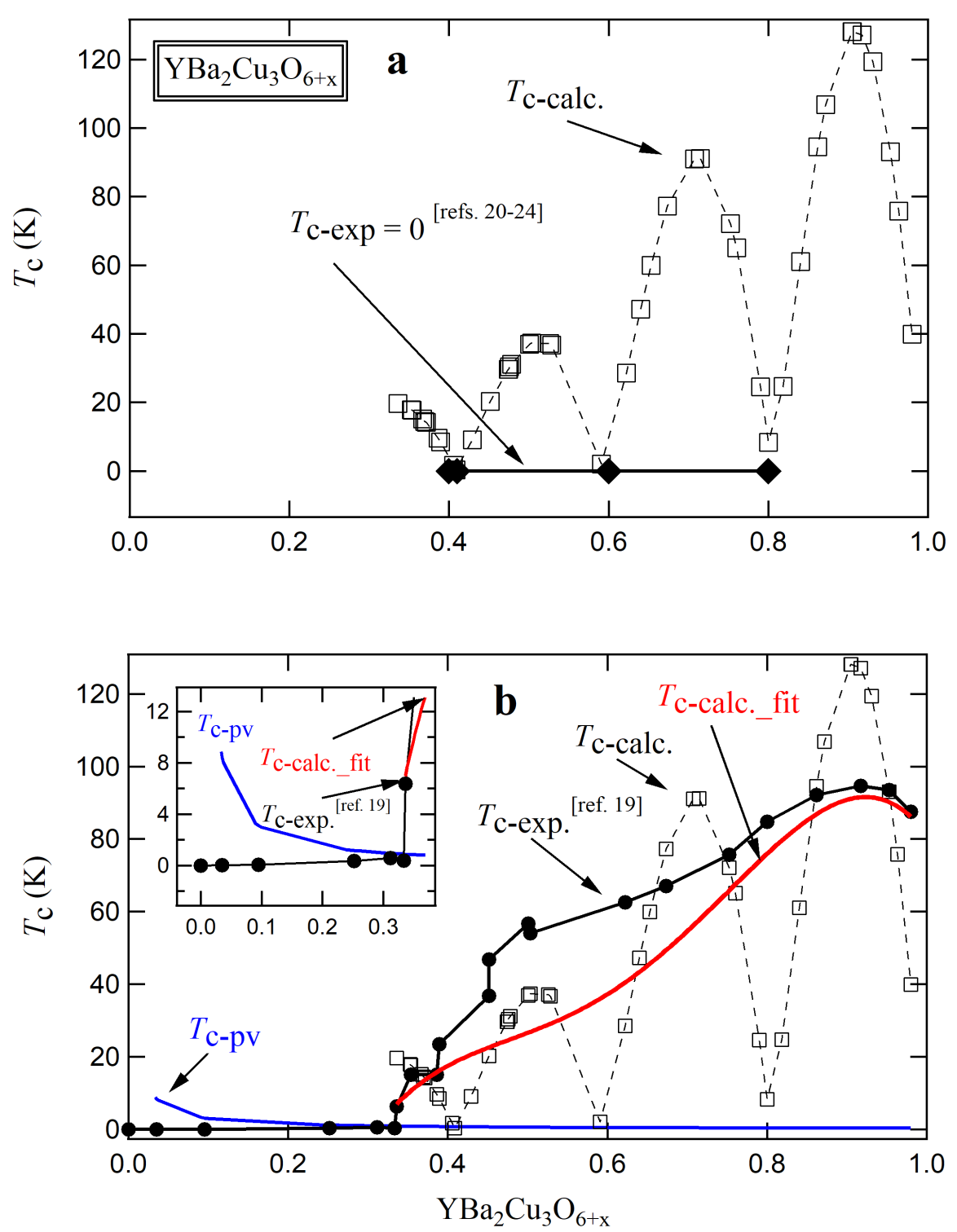

Figure S3: Transition temperature $\left(T_{c}\right)$ phase diagrams of superconducting and non-superconducting $\mathrm{YBa}_{2} \mathrm{Cu}_{3} \mathrm{O}_{6+x}$. Plots $T_{c-e x p}, T_{c-P V}, T_{c-c a l c}$ and $T_{c-\text { calc-fit }}$ represent transition temperatures measured experimentally, ${ }^{19-24}$ estimated from equation (1), calculated from equation (2), and the least-squares fit of $T_{c-c a l c}$ with a 5 th order polynomial, respectively.(a) Transition temperature versus oxygen intercalation for $T_{c-c a l c}$ and experimentally determined non-superconducting phases of $T_{c-e x p} .{ }^{20-24}(\mathbf{b})$ Transition temperature versus oxygen intercalation for $T_{c-\text { calc }}$ and experimentally determined superconducting phases of $T_{c-\text { exp }} .{ }^{19}$ Inset shows onset of superconductivity at low oxygen intercalation, $x<0.4$. 
Among all high temperature superconductors, $\mathrm{YBa}_{2} \mathrm{Cu}_{3} \mathrm{O}_{6+x}$ (YBCO) provides an unparalleled perspective to high $T_{c}$ superconductivity. This is because regarding doping, the landscape of high temperature superconductors is dominated by 2 types. The first are materials doped under particle exchange (e.g., cation-substituted $\mathrm{Bi}_{2} \mathrm{Sr}_{2} \mathrm{Ca}_{1-x} \mathrm{Lu}_{x} \mathrm{Cu}_{2} \mathrm{O}_{8-\delta}$ ). ${ }^{25}$ The second are metallic compounds which are then doped to vary their superconducting properties (e.g., iodine doped $\left.\mathrm{BSCCO}, \mathrm{IBi}_{2} \mathrm{Sr}_{2} \mathrm{CaCu}_{2} \mathrm{O}_{8}\right) \cdot{ }^{26} \mathrm{YBCO}$ is unique because it is an oxygen intercalate whose superconductivity emerges from an insulator, $\mathrm{YBa}_{2} \mathrm{Cu}_{3} \mathrm{O}_{6} .{ }^{19}$ Therefore, YBCO fits the premise of the main manuscript.

YBCO's calculated phase diagram shown above in Figure S3a displays regions where little to no superconductivity occurs. These regions are near $x=0.4,0.6$, and 0.8 . Experimentally, these specific regions were also observed to be non-superconducting. The $x=0.4$ phase is semiconducting, ${ }^{20,21}$ however, it superconducts if quenched after growth to minimize the insulating effects due to oxygen vacancy ordering. ${ }^{27} \mathrm{~A}$ similar observation was made for the $x=0.6$ phase as well. ${ }^{22,23}$ The phase $x=0.8$ also exhibits insulating behavior, mainly attributed to its microstructural properties. ${ }^{24}$ In the end, the entire phase diagram of YBCO has been shown to be a competition between superconductivity and Anderson localization as oxygen intercalation increases. ${ }^{28}$ Therefore, the above observations possibly give further credence to the ideas developed within the main report, most probably due to the initial use of steady state variables and conditions, which innately disregard time-dependent effects such as quenching.

The phase diagram derived from experiment for $T_{c-e x p}>0$, shown in Figure S3b, does not exactly coincide with the calculated curve. However, a general increase in $T_{c}$ within both curves is evident with increasing oxygen intercalation. Fitting the calculated curve to a 5th order polynomial by regression, shown in Figure S3b, yields a curve which is like the expected in terms on $T_{c}$ and $\mathrm{d} T_{c} / \mathrm{d} x$. It is important to note the plot of the "ideal" onset equation $\left(P V=x N K_{B} T_{c-P V} \rightarrow\right.$ blue curve in Figure S3's inset) clearly passes through the onset of superconductivity within the graph generated from experimental data, as generally 
shown to be expected for intercalated insulators throughout the main report.

\section{References}

(1) Morosan, E.; Zandbergen, H. W.; Dennis, B. S.; Bos, J. W. G.; Onose, Y.; Klimczuk, T.; Ramirez, A. P.; Ong, N. P.; Cava, R. J. Superconductivity in $\mathrm{Cu}_{x} \mathrm{TiSe}_{2}$. Nature Physics 2006, 2, 544-550.

(2) Li, S. Y.; Wu, G.; Chen, X. H.; Taillefer, L. Single-gap s-wave superconductivity near the charge-density-wave quantum critical point in $\mathrm{Cu}_{x} \mathrm{TiSe}_{2}$. Physical Review Letters 2007, 99, 107001.

(3) Morosan, E.; Li, L.; Ong, N. P.; Cava, R. J. Anisotropic properties of the layered superconductor $\mathrm{C}_{0.07} \mathrm{TiSe}_{2}$. Physical Review B 2007, 75, 104505.

(4) Zhao, J. F.; Ou, H. W.; Wu, G.; Xie, B. P.; Zhang, Y.; Shen, D. W.; Wei, J.; Yang, L. X.; Dong, J. K.; Arita, M.; Namatame, H.; Taniguchi, M.; Chen, X. H.; Feng, D. L. Evolution of the Electronic Structure of $1 T-\mathrm{Cu}_{x} \mathrm{TiSe}_{2}$. Physical Review Letters 2007, 99, 146401.

(5) Kulbachinskii, V.; Bulychev, B.; Kytin, V.; Krechetov, A.; Tarasov, V.; Konstantinova, E.; Velikodnyi, Y.; Muravlev, Y.; Zoteev, A. Magnetic and structural anomalies of $\mathrm{Na}_{n} \mathrm{C} 60$ ( $\left.\mathrm{n}=2,3\right)$. Open Physics 2010, 8, 101-112.

(6) Holczer, K.; Klein, O.; Grüner, G.; Thompson, J. D.; Diederich, F.; Whetten, R. L. Critical magnetic fields in the superconducting state of $\mathrm{K}_{3} \mathrm{C}_{60}$. Physical Review Letters 1991, 67, 271-274.

(7) Ramirez, A. P.; Kortan, A. R.; Rosseinsky, M. J.; Duclos, S. J.; Mujsce, A. M.; Haddon, R. C.; Murphy, D. W.; Makhija, A. V.; Zahurak, S. M.; Lyons, K. B. Isotope effect in superconducting $\mathrm{Rb}_{3} \mathrm{C}_{60}$. Physical Review Letters 1992, 68, 1058-1060. 
(8) Ihara, Y.; Alloul, H.; Wzietek, P.; Pontiroli, D.; Mazzani, M.; Riccò, M. NMR Study of the Mott Transitions to Superconductivity in the Two $\mathrm{Cs}_{3} \mathrm{C}_{60}$ Phases. Physical Review Letters 2010, 104, 256402.

(9) Ganin, A. Y.; Takabayashi, Y.; Khimyak, Y. Z.; Margadonna, S.; Tamai, A.; Rosseinsky, M. J.; Prassides, K. Bulk superconductivity at $38 \mathrm{~K}$ in a molecular system. Nature Materials 2008, 7, 367-371.

(10) Slater, J. C. Atomic Radii in Crystals. The Journal of Chemical Physics 1964, 41, 3199-3204.

(11) Clementi, E.; Raimondi, D. L.; Reinhardt, W. P. Atomic Screening Constants from SCF Functions. II. Atoms with 37 to 86 Electrons. The Journal of Chemical Physics 1967, 47, 1300-1307.

(12) Mitsuhashi, R.; Suzuki, Y.; Yamanari, Y.; Mitamura, H.; Kambe, T.; Ikeda, N.; Okamoto, H.; Fujiwara, A.; Yamaji, M.; Kawasaki, N.; Maniwa, Y.; Kubozono, Y. Superconductivity in alkali-metal-doped picene. Nature 2010, 464, 76-79.

(13) Wang, X. F.; Liu, R. H.; Gui, Z.; Xie, Y. L.; Yan, Y. J.; Ying, J. J.; Luo, X. G.; Chen, X. H. Superconductivity at $5 \mathrm{~K}$ in alkali-metal-doped phenanthrene. Nature Communications 2011, 2, 507 .

(14) Moroshkin, P.; Hofer, A.; Ulzega, S.; Weis, A. Spectroscopy of Rb 2 dimers in solid 4He. Physical Review A 2006, 74.

(15) Moroshkin, P.; Hofer, A.; Lebedev, V.; Weis, A. Spectroscopy of $\mathrm{Cs}_{2}$, RbCs, and $\mathrm{Rb}_{2}$ in solid 4He. The Journal of Chemical Physics 2010, 133, 174510.

(16) Hesper, $\mathrm{R}$. The influence of surfaces and interfaces on the properties of $\mathrm{C}_{60}$ compounds. 2000 , 
(17) Bensebaa, F.; Xiang, B.; Kevan, L. Electron spin resonance of alkali-metal-doped fullerenes. Journal of Physical Chemistry 1992, 96, 10258-10261.

(18) Irons, S. H.; Liu, J. Z.; Klavins, P.; Shelton, R. N. Magnetic properties of superconducting $\mathrm{K}_{3} \mathrm{C}_{60}$ and $\mathrm{Rb}_{3} \mathrm{C}_{60}$ synthesized from large single-crystal fullerenes. Physical Review B 1995, 52, 15517-15521.

(19) Liang, R.; Bonn, D. A.; Hardy, W. N. Evaluation of $\mathrm{CuO}_{2}$ plane hole doping in $\mathrm{YBa}_{2} \mathrm{Cu}_{3} \mathrm{O}_{6+x}$. Physical Review B 2006, 73, 180505.

(20) Ottaviani, G.; Nobili, C.; Nava, F.; Affronte, M.; Manfredini, T.; Matacotta, F. C.; Galli, E. Oxygen in-diffusion processes in tetragonal $\mathrm{YBa}_{2} \mathrm{Cu}_{3} \mathrm{O}_{7-x}$ oxide. Physical Review B 1989, 39, 9069-9073.

(21) Moodenbaugh, A. R.; Fischer, D. A. Hole state density and superconductivity in oxygen-deficient $\mathrm{La}_{2-x} \mathrm{Sr}_{x} \mathrm{CuO}_{4-y}$ and $\mathrm{YBa}_{2} \mathrm{Cu}_{3} \mathrm{O}_{7-\delta}$. Physica C: Superconductivity 1994, 230, 177-183.

(22) Forró, L.; Petravić, M.; Leontić, B. Hall-effect of the high $\mathrm{T}_{c}$ superconductors Y-BaCu-O and Gd-Ba-Cu-O. Solid State Communications 1988, 65, 1355-1358.

(23) Yan, Y.; Blanchin, M.-G.; Picard, C.; Gerdanian, P. Oxygen ordering in $\mathrm{YBa}_{2} \mathrm{Cu}_{3} \mathrm{O}_{z}(7 \geq z>6)$ superconductors with equilibrium defect structure at room temperature. Journal of Materials Chemistry 1993, 3, 603-607.

(24) Caignaert, V.; Hervieu, M.; Wang, J.; Desgardin, G.; Raveau, B.; Boterel, F.; Haussonne, J. M. $\mathrm{YBa}_{2} \mathrm{Cu}_{3} \mathrm{O}_{6.8}$ : an oxygen-rich "123"-type phase with tetragonal symmetry. Physica C: Superconductivity 1990, 170, 139-152.

(25) Koike, Y.; Iwabuchi, Y.; Hosoya, S.; Kobayashi, N.; Fukase, T. Correlation between Tc and hole concentration in the cation-substituted $\mathrm{Bi}_{2} \mathrm{Sr}_{2} \mathrm{CaCu}_{2} \mathrm{O}_{8+\delta}$. Physica C: Superconductivity 1989, 159, 105-110. 
(26) Xiang, X.-D.; McKernan, S.; Vareka, W. A.; Zettl, A.; Corkill, J. L.; Barbee, T. W.; Cohen, M. L. Iodine intercalation of a high-temperature superconducting oxide. Nature $1990,348,145-147$.

(27) Jones, E. C.; Norton, D. P.; Christen, D. K.; Lowndes, D. H. Anomalous T ${ }^{3}$ Inverse Hall Mobilities Observed in $\mathrm{Sr}_{1-x} \mathrm{CuO}_{2}$ and $\mathrm{Sr}_{0.9} \mathrm{Nd}_{0.1} \mathrm{CuO}_{2}$ Infinite-Layer Thin Films. Physical Review Letters 1994, 73, 166-169.

(28) Matsushita, A.; Oguchi, T.; Kimura, K.; Matsumoto, T.; Hatano, T.; Ogawa, K.; Takayanagi, S. Low Temperature Specific Heat and Electrical Resistivity in Orthorhombic $\mathrm{YBa}_{2} \mathrm{Cu}_{3} \mathrm{O}_{6.8}$ and Tetragonal $\mathrm{YBa}_{2} \mathrm{Cu}_{3} \mathrm{O}_{6.0}$. Japanese Journal of Applied Physics 1987, 26, L1953. 